Antoine Ménoret Jacques Le Pendu

\section{ADRESSE}

A. Ménoret : chercheur post-doctoral. J. Le Pendu: directeur de recherche à l'Inserm. CJF Inserm 90-11, Institut de biologie, 9, quai Moncousu, 44035 Nantes Cedex 01, France.

\title{
Protéines de choc thermique et antigènes tumoraux
}

Les protéines de choc thermique (HSP), molécules chaperons, s'associent à des peptides ou à des protéines mal repliées. Leur rôle d'accepteur de peptides et de transporteurs de ces peptides vers les molécules du complexe majeur d'histocompatibilité, l'existence de réponses immunitaires constitutives anti-HSP, suggèrent une participation importante de ces protéines à l'immunité cellulaire. Dans les cellules tumorales, où elles sont souvent surexprimées, les HSP s'associent à des protéines « anormales » potentiellement antigéniques. L'immunisation de souris contre des préparations de HSP purifiées à partir de certaines tumeurs protège les animaux de l'implantation de ces tumeurs, à la condition que HSP et peptides tumoraux soient restés associés. Cependant, la nature de ces peptides est encore inconnue et les mécanismes de la réponse antitumorale relayée par les HSP partiellement incompris.

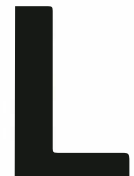

es protéines de choc thermique ou heat shock proteins (HSP) appartiennent à une famille multigénique très conservée au cours de l'évolution et dont la synthèse est stimulée en réponse à divers stress, comme le choc thermique, qui accélèrent la dénaturation des protéines. Différents groupes de HSP ont été définis en fonction de leur masse moléculaire apparente (hspl10, hsp90, hsp70, hsp60, hsp20 et l'ubiquitine). C'est sur la base d'homologies de séquence que les GRP ou glucose regulated proteins, grp94, grp78 (BiP) et grp58, localisées dans le réticulum endoplasmique, ont été classées dans la famille des HSP. Les protéines de choc thermique sont des chaperons moléculaires. C'est-àdire que leur seule fonction est de se fixer sur les protéines qui ne sont pas dans leur structure native : ceux de la famille hsp70 se fixent sur les protéines totalement dénaturées, tandis que ceux de la famille hsp60 (ou GroEl, GroEs) se fixent sur les protéines partiellement repliées à l'état de molten globule [1]. Chez les eucaryotes, les différentes HSP assurent un rôle de chaperon en fonction de leur localisation dans les organites intracellulaires. Ainsi le récepteur des glucocorticoïdes est complexé aux hsp90, hsp70 et hsp65 dans le noyau [2]. Les protéines cytosoliques se lient à l'hsc 70 (heat shock cognate protein $70 \mathrm{kDa}$ ) au cours de leur synthèse et à l'hsp70 en cas de dénaturation. Certains auteurs ont, en outre, suggéré que les HSP pourraient être présentes sur la membrane plasmique. Cependant, ce point reste controversé.

Sur le plan immunologique, les HSP 


\section{RÉFÉRENCES}

1. Hendrick JP, Hartl FU. Molecular chaperone functions of heat-shock proteins. Annu Rev Biochem 1993; 62 : 349-84.

2. Pratt WB. The role of heat shock proteins in regulating the function, folding, and trafficking of the glucocorticoid receptor. I Biol Chem 1993; 268 : 21455-8.

3. Jacquier-Sarlin MR, Polla BS. Protéines de stress: soi, non-soi et réponse immune. médecine/sciences 1993 ; 10 : 31-42.

4. Srivastava PK, DeLeo AB, Old LJ. Tumor rejection antigens of chemically induced sarcomas of inbred mice. Proc Natl Acad Sci USA 1986; 83: 3407-11.

5. Ullrich SJ, Robinson EA, Law LW, Willingham M, Appella E. A mouse tumorspecific transplantation antigen is a heat shock-related protein. Proc Natl Acad Sic USA 1986; 83: 3121-5.

6. Maki RG, Old LJ, Srivastava PK. Human homologue of murine tumor rejection antigen gp96: 5'-regulatory and coding regions and relationship to stress-induced proteins. Proc Natl Acad Sci USA 1990 ; 87 : 5658-62.

7. Yufu Y, Nishimura J, Nawata H. High constitutive expression of heat shock protein 90a in human acute leukemia cells. Leukemia Res 1992; 16: 597-605.

8. Ferrarini M, Heltai S, Zocchi MR, Rugarli C. Unusual expression and localisation of heat-shock proteins in human tumor cells. Int J Cancer 1992; 51 : 61.3-9.

9. Strahler JR, Kuick R, Eckerson C, et al. Identification of two related markers for common acute lymphoblastic leukemia as heat shock proteins. J Clin Invest 1990 ; 85 : 200-7.

10. Li $\mathrm{Z}$, Srivastava $\mathrm{PK}$. Tumor rejection antigen gp96/grp94 is an ATPase: implication for protein folding and antigen presentation. EMBO J 1993; 12 : 314.3-51.

11. Blachère NE, Udono $H$, Janetzki $S, L i$ Z, Heike M, Srivastava P. Heat shock protein vaccines against cancer. I Immunothe rapy 1993 ; $14: 352-6$.

12. Udono H, Levey D, Srivastava PK Cellular requirement for tumor specific immunity elicited by heat shock proteins : tumor rejection antigen gp96 primes naive CD8+ $\mathrm{T}$ cells in vivo. Proc Nall Acad Sci USA $1994 ; 91: 3077-81$ constituent des antigènes puissants pour l'activation lymphocytaire. Ia stimulation des lymphocytes T spécifiques des HSP pourrait amplifier la réponse immunitaire et participer à la surveillance immune, à l'élimination des cellules infectées (cellules $\mathrm{T}$ cytotoxiques), à la protection (immunité de vaccination), mais aussi à des phénomènes d'autoimmunité par mimétisme moléculaire entre les HSP des pathogènes et celles de l'hôte (cellules $\mathrm{T}$ auxiliaires), enfin à la protection précoce au niveau des épithéliums (cellules T $\gamma \delta$ ) [3].

\section{Rejet de tumeurs relayé par les HSP}

\section{Gp96 et hsp90}

Depuis des dizaines d'années, les immunologistes sont à la recherche d'antigènes capables d'induire le rejet de tumeurs. Le concept de l'existence de telles molécules appelées TATA (transplantation associated tumor antigen) ou TRA (tumor rejection antigen) repose sur des expériences d'immunisation anti-tumorale réalisées chez l'animal. Dans certains modèles, une tumeur greffée en sous-cutané sera rejetée spécifiquement par le système immunitaire de l'hôte syngénique si l'animal a été préalablement immunisé avec des cellules, irradiées ou fractionnées par voie biochimique, provenant de la même tumeur. Il y a quelques années, deux équipes indépendantes, celle de Old et celle d'Apella ont cru caractériser deux de ces antigènes tumoraux dans le modèle de sarcome murin Meth A. Il s'agissait respectivement d'une glycoprotéine de 96 kDa (gp96) [4] et d'un dimère protéique de 84 et $86 \mathrm{kDa}$ (p84/86) [5]. Chacune de ces molécules, purifiée à partir d'extraits cellulaires, permettait d'immuniser des animaux contre la tumeur Meth A. I a protection conférée par ces immunisations était spécifique à la tumeur Meth A et ne concernait pas d'autres tumeurs syngéniques. Ces deux molécules appartiennent à la famille des protéines de choc thermique. Elles présentent une forte homologie entre elles et avec les autres HSP [6]. La p84/86 s'est avérée être l'hsp 90 localisée dans le cytosol. La gp96 est son équivalent moléculaire localisé dans le réticulum endoplasmique et se trouve correspondre à la grp94/100 (glucose regulated protein $94 / 100 \mathrm{kDa}$ ), également nommée endoplasmine ou ERpp99. Elle présente aussi une forte homologie avec l'hspl08 [6]. I.e clonage et le séquençage de ces molécules ne révéla aucune mutation capable d'expliquer leurs propriétés antigéniques. Les HSP étant des molécules excessivement conservées, leur rôle d'antigène tumoral spécifique paraissait intrigant mais pouvait être lié, soit à leur surexpression [7], soit à leur localisation anormale [8] dans les cellules tumorales. Ces anomalies d'expression sont telles que l'hsp27 peut même être considérée comme un marqueur de la leucémie aiguë lymphoblastique [9].

Cependant, comme les HSP ont la capacité de se lier à différentes molécules, en particulier à des peptides, il a été proposé qu'elles ne seraient pas antigéniques par ellesmêmes, mais qu'elles agiraient comme porteurs de peptides antigéniques [6]. Depuis, une série d'observations faites sur la gp96 sont venues étayer cette hypothèse: (1) des peptides peuvent être associés à la gp96 [10], (2) la gp96 est une ATPase [10], la gp96 s'associe avec les molécules du complexe majeur d'histocompatibilité (CMH) de classe I pendant l'assemblage CMH I/ $\beta 2$ microglobuline/peptide [10], (3) des lymphocytes T cytotoxiques (CTL) dirigés contre des antigènes du virus de l'influenza peuvent être engendrés par immunisation avec la gp96 isolée de cellules infectées par ce virus [11], (4) l'immunisation avec des complexes gp96/peptides solubles stimule spécifiquement des lymphocytes T CD8+ [12].

\section{Hsp70}

L'immunisation de souris avec des molécules d'hsp70 du fibrosarcome Meth A, purifiées à homogénéité, montre que les propriétés de la gp96 sont applicables à d'autres protéines de stress. En effet, après immunisation, ces souris greffées avec le sarcome Meth A sont capables d'inhiber la croissance tumorale 


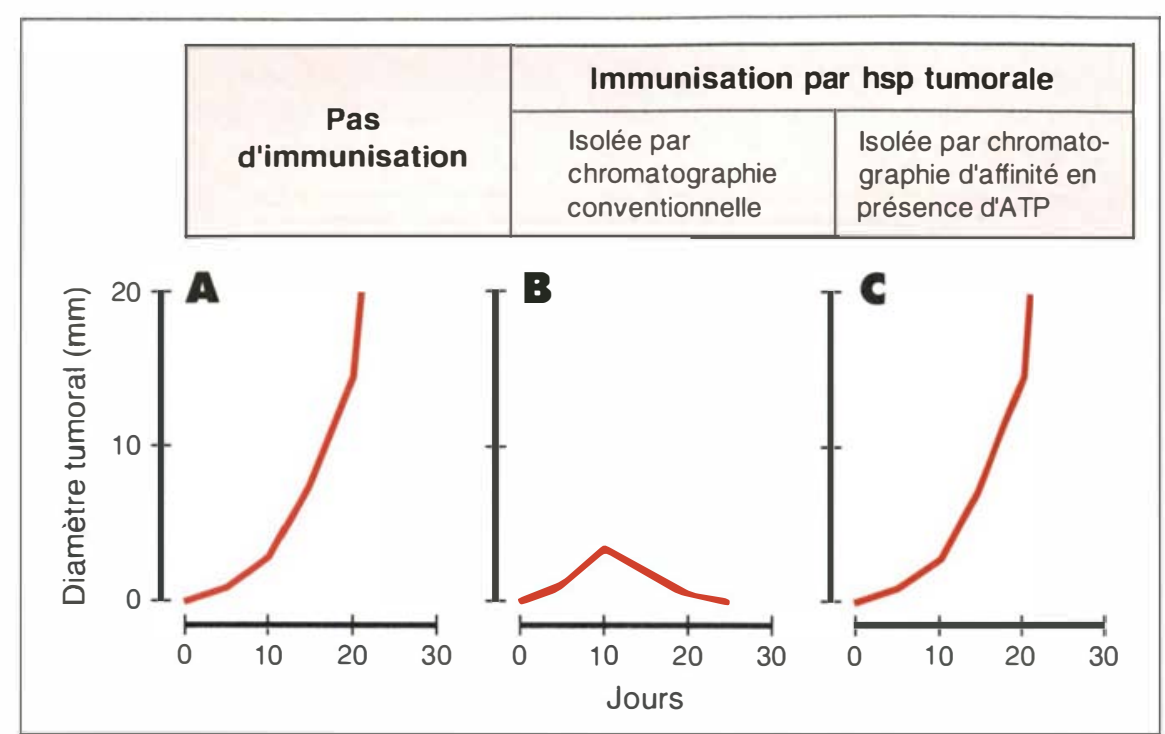

Figure 1. Immunogénicité de I'hsp70 purifiée et mise en évidence de la sensibilité à I'ATP de I'association hsp70/peptides. (A) La tumeur Meth A progresse chez les souris syngéniques non immunisées. (B) Les souris sont cependant protégées par immunisation contre l'hsp70 tumorale purifiée par chromatographie conventionnelle, c'est-à-dire dans des conditions qui maintiennent l'association entre I'HSP et des peptides potentiellement antigéniques. (C) En revanche, les souris ne sont plus protégées lorsqu'elles sont immunisées par l'hsp70 tumorale purifiée par chromatographie d'affinité en présence d'ATP qui dissocie les complexes hsp70/peptides. C'est donc un peptide associé à l'hsp70 qui permet de développer l'immunité contre le sarcome Meth A [13].

de façon significative et peuvent, éventuellement, rejeter la tumeur en fonction de la dose d'hsp70 utilisée [13]. L’immunogénicité de cette protéine ubiquitaire, présente aussi bien dans le tissu normal que dans les tumeurs, fut testée après isolement et purification à partir de ces tissus. Aucun effet de protection tumorale de l'hsp70 dérivée de tissus normaux ne fut détecté. A l'inverse, l'immunité engendrée par vaccination avec l'hsp70 issue de Meth A est efficace contre le développement tumoral (figure 1). Cette protection est spécifique de cette tumeur: des souris immunisées avec l'hsp70 de Meth A n'inhibent pas le développement d'autres tumeurs induites par le méthylcholanthrène (CM, CMS5) dont l'antigénicité est différente. Les molécules d'hsp70, préparées à partir de tissus normaux et du sarcome Meth A, sont donc également distinctes au point de vue antigénique [13]. L'hsp70 est fortement conservée et non polymorphique, mais il se pourrait que les $\mathrm{m} / \mathrm{s} \quad n^{\circ}$ 6-7 vol. 10, juin-juillet 94
d'ATP forment un mélange complexe à l'analyse par chromatographie en phase inverse. Ces peptides proviennent probablement de protéines cellulaires en cours de synthèse, ou soumises à une dégradation protéolytique, ou encore mutées. Leur identification permettra de définir la nature des antigènes impliqués dans le rejet tumoral.

\section{Relation avec les molécules du CMH}

Une étude de la structure secondaire de l'hsp70, à partir d'une séquence consensus de son domaine de liaison peptidique, a été réalisée à partir de 33 séquences d'hsp70 d'origines diverses, dans le but de mieux comprendre le mécanisme d'interaction moléculaire développé par cette famille de protéines. La séquence secondaire déduite présente un motif très ressemblant à celui de HLA A2 humain, des résidus hydrophobes de l'hsp70 s'alignant avec ceux de la poche à peptide de la molécule HIA [16]. Cette similitude topologique laisse supposer que les deux familles de molécules ont une potentialité équivalente de liaison des peptides et qu'elles dérivent probablement d'un ancêtre commun. La nature des peptides pouvant se lier aux HSP a été testée en mesurant leur capacité à stimuler l'activité ATPasique de la grp78/BiP. La stimulation optimale est obtenue avec des heptapeptides préférentiellement hydrophobes, correspondant à des zones internes des protéines lorsque celles-ci sont correctement repliées [15]. Un travail récent de Blond-ElGuindi et al. a montré que la grp78/BiP fixait préférentiellement des peptides contenant des acides aminés aromatiques et hydrophobes en alternance avec d'autres acides aminés [17].

La présentation d'antigènes par les molécules du CMH de classe II peut être bloquée par des anticorps reconnaissant une protéine appartenant à la famille des hsp70 : la PBP 72/74 (peptide-binding protein 72/74 $\mathrm{kDa}$ ) [14]. Cette HSP jouerait un rôle dans la formation des complexes des molécules du CMH de classe II. Elle est localisée au niveau 


\section{RÉFÉRENCES}

13. Udono $\mathrm{H}$, Srivastava PK. Heat shock protein 70-associated peptides elicit specific cancer immunity. J Exp Med 1993; 178 : 1391-6.

14. Lakey EK, Margoliash E, Pierce SK. Identification of a peptide binding protein that plays a role in antigen presentation. Proc Natl Acad Sci USA 1987; 84 : 1659-63.

15. Flynn GC, Polh J, Flocco MT, Rothman JE. Peptide-binding specificity of the molecular chaperone BiP. Nature 1991; 353 : 726-30.

16. Rippman F, Taylor WR, Rothbard JB, Green NM. A hypothetical model for the peptide binding domain of $\mathrm{hsp} 70$ ba $\mathrm{d}$ on the peptide binding domain of HIA. EMBO J 1991 ; 10 : 1053-9.

17. Blond-ElGuindi S, Cwirla SE, Dower WJ, Lipshultz RJ, Sprang SR, Sambrook JF, Gething $\mathrm{MJH}$. Affinity panning of a library of peptides displayed on bacteriophages reveals the binding specificity of BIP. Cell 1993 ; 75 : 717-28.

18. VanBuskirk AM, DeNagel DC, Guagliardi LE, Brodsky FM, Pierce SK. Cellular and subcellular distribution of pbp72/74, a peptide-binding protein that plays a role in antigen processing. J Immunol 1991; 146 : 500-6.

19. DeNagel DC, Pierce SK. A case for chaperones in antigen processing. Immunol Today 1992 ; 13 : 86-9.

20. Srivastava PK. Peptide-binding heat shock proteins in the endoplasmic reticulum: role in immune response to cancer and in antigen presentation. Adv Cancer Res 1993 ; 62 : 153-77.

21. Harada M, Matsuzaki G, Yoshikai Y, et al. Autoreactive and heat shock protein 60-recognizing CD4+ T-cells show antitumor activity against syngeneic fibrosarcoma. Cancer Res 1993; 53: 106-11.

22. Debrick JE, Campbell PA, Staerz UD. Macrophages as accessory cells for class I MHC-restricted immune responses. J Immunol 1991; 147: 2846-51

23. Hosmalin A, Kumar S, Barnd D, et al. Immunisation with soluble protein-pulsed spleen cells induces class I-restricted cytotoxic $T$ lymphocytes that recognize immunodominant epitopic peptides from Plasmo dium falciparum and HIV-1. J Immunol 1992 ; 149: 1311-8.

24. Bensaude O. Protéines de choc thermique, transport des protéines dans le noyau et oncogenèse. médecine/sciences $1992 ; 8: 710-3$.

25. Davidof AM, Iglehart JD, Marks JR. Immune response to p53 is dependent upon p53/HSP70 complexes in breast cancers. Proc Natl Acad Si USA 1992; 89 . des vésicules endosomiques, de la lumière du réticulum endoplasmique, et est aussi observée au niveau de la membrane plasmique [18]. La PBP72/74, qui possède une fonction ATPasique, faciliterait le " chargement " de peptides dans le CMH de classe II en modifiant la conformation de l'ensemble moléculaire et/ou transporterait des peptides, des compartiments cellulaires où ils sont produits, jusqu'au compartiment contenant le CMH de classe II $[3,19]$.

Il a récemment été aussi proposé que la gp96, protéine très abondante du réticulum endoplasmique, s'associerait avec des peptides qu'elle transférerait sur la molécule du CMH de classe I [20]. Les HSP ne seraient donc pas des molécules présentatrices d'antigènes en lieu et place des molécules du $\mathrm{CMH}$, mais elles faciliteraient la formation et la mise en place d'une conformation correcte des complexes CMH/peptides.

\section{Mécanismes possibles de la réponse antitumorale relayée par les HSP}

Le mécanisme immunitaire de la réponse antitumeur engendrée par l'immunisation avec une HSP tumorale fait donc probablement intervenir plusieurs types cellulaires dépendant d'une régulation complexe. Comme il est schématisé dans la figure 2, une préparation de gp96 ou d'hsp70 purifiée à partir du sarcome Meth A, potentiellement associée à un ou plusieurs antigènes tumoraux, pourrait être reconnue par des immunoglobulines solubles ou présentes à la surface de lymphocytes B auto-immuns. Les complexes HSP/peptides seraient alors internalisés et présentés par le CMH de classe II à des lymphocytes CD4+. Les peptides présentés seraient de trois types possibles: (1) des fragments d'HSP induisant une réponse du type anti-HSP, réprimée par un réseau de lymphocytes auto-immuns. Il existe en effet des lymphocytes CD4+ auto-réactifs spécifiques de l'hsp60, possédant une activité antitumorale, à la fois cytostatique et d'induction de lymphocytes $T$ cyto- toxiques [21] ; (2) des fragments de peptides, initialement associés à la gp96, provenant de protéines du soi et n'induisant pas de réponse immune; (3) des fragments d'autres peptides, initialement associés à l'HSP, provenant de protéines "anormales" induisant une réponse antitumorale spécifique. Si le rôle des lymphocytes B n'est actuellement qu'hypothétique, en revanche celui des macrophages a été montré par des expériences de déplétions fonctionnelles annulant entièrement l'effet de l'immunisation par la gp96 tumorale [ I2]. L'internalisation du complexe gp96/antigène tumoral induit, par un mécanisme probablement équivalent à celui décrit pour les lymphocytes $\mathrm{B}$, une réponse antitumorale de lymphocytes CD4+, mais également de lymphocytes CD8+. Il est généralement admis que des molécules solubles exogènes induisent une réponse des cellules CD4+ dépendant d'une présentation de peptides par les molécules du $\mathrm{CMH}$ de classe II, alors que des protéines endogènes sont présentées dans le contexe des molécules du $\mathrm{CMH}$ de classe I et induisent une réponse des cellules CD8+. Dans le cas particulier de la réponse CD8+ induite par immunisation avec la gp96 exogène soluble, Srivastava propose que des récepteurs présents à la surface des macrophages fixeraient les HSP pour les diriger ensuite vers une voie de présentation indépendante du compartiment lysosomial, permettant une présentation dans le contexte du CMH de classe I [20]. Il est également possible que cette reconnaissance par les macrophages soit réalisée par le récepteur du fragment $F_{C}$ des immunoglobulines, fixant et internalisant des immunoglobulines autoimmunes anti-HSP elles-mêmes liées au complexe HSP/antigène tumoral (figure 2). La présentation d'antigènes solubles exogènes par les macrophages via une voie de présentation endogène conduisant à une restriction par le $\mathrm{CMH}$ de classe I a déjà été suggérée dans deux autres systèmes [22, 23].

Il est très important de noter que des doses extrêmement faibles d'hsp70, d'hsp90, ou de gp96 (de l'ordre de quelques $\mu \mathrm{g}$ ) suffisent à 


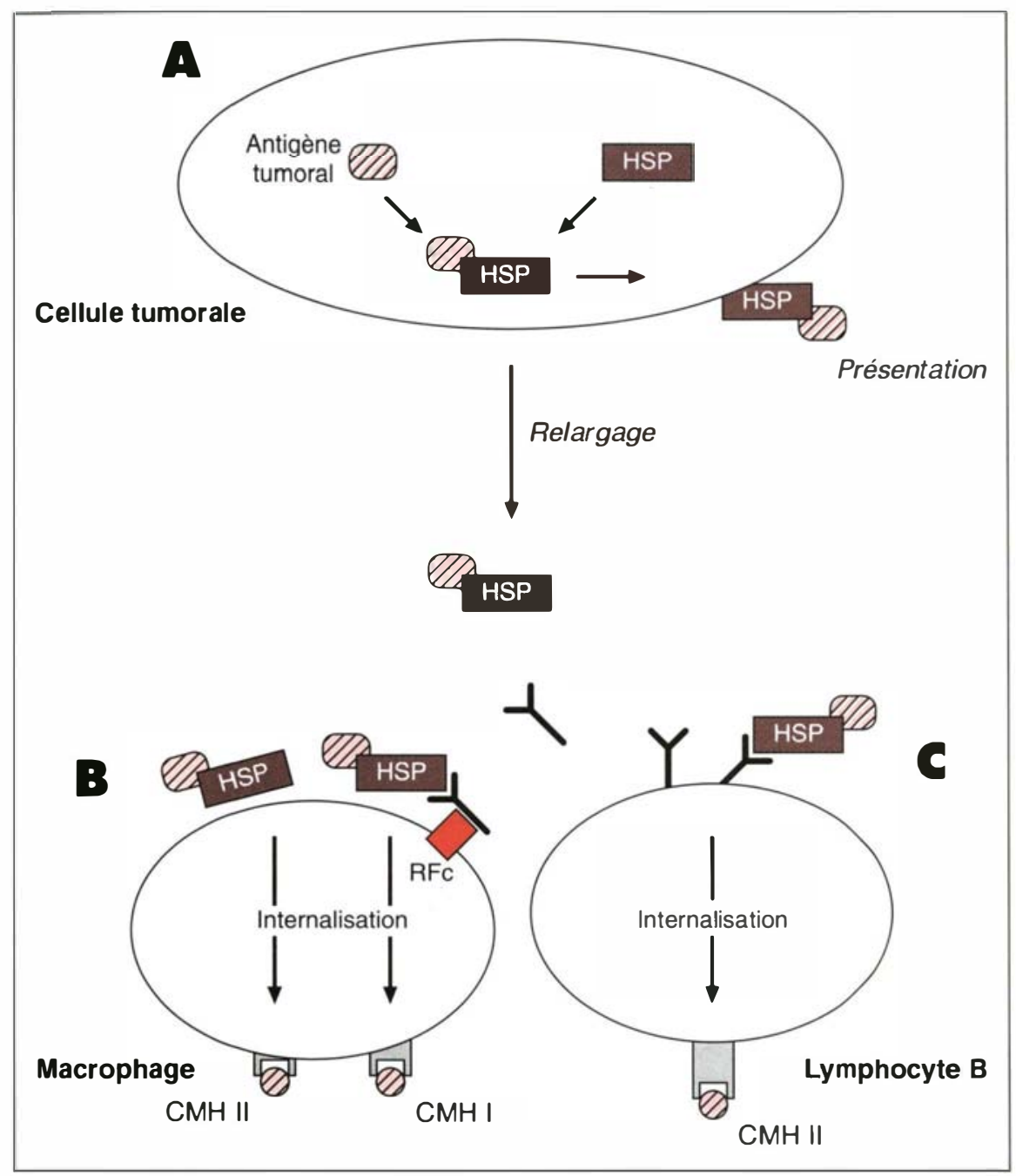

Figure 2. Mécanismes possibles du rôle des HSP dans l'immunisation antitumorale. (A) Les similitudes entre HSP et antigènes du CMH laissent supposer que les HSP, éventuellement présentes à la membrane des cellules tumorales en situation de stress, pourraient agir comme molécules présentatrices $d^{\prime}$ antigènes. Par ailleurs, le relargage de complexes peptides/HSP potentialiserait la présentation du peptide antigénique au système immunitaire. Pour cela plusieurs mécanismes peuvent être envisagés. (B) Le complexe pourrait être internalisé par des macrophages, via un mécanisme encore inconnu [20], après quoi le peptide serait transferé sur des molécules du CMHI ou CMHII. Ce même complexe pourrait aussi être reconnu par une immunoglobuline auto-immune anti-HSP, puis fixé par le récepteur de la fraction Fc des immunoglobulines exprimé à la surface du macrophage, enfin internalisé vers une voie de présentation par le CMHI. (C) Les lymphocytes B auto-immuns anti-HSP capturant le complexe HSP/antigène tumoral par leurs immunoglobulines de surface pourraient internaliser l'antigène tumoral et le présenter sous la forme de peptides par leur CMH II. La présentation dans le contexte du CMH II stimulerait des lymphocytes CD4+ alors que les lymphocytes CD8+ antitumeurs seront activés après présentation de peptides dans le contexte du CMHI.

$m / s \quad n^{\circ} 6-7$ vol. 10 , juin.juillet 94 induire le rejet des tumeurs $[4,5]$, et que des doses plus importantes n'ont pas d'effet ou conduisent à une facilitation de la croissance tumorale, voire même à la mort de l'animal [4]. Le mécanisme mis en œuvre ici est donc réglé très finement puisqu'il n'est efficace qu'à dose très précise d'immunogène. L'immunisation avec une HSP purifiée de tumeur permettrait donc dans un premier temps, d'amplifier une réponse auto-immune préexistante pour, dans un deuxième temps, déclencher une réponse immune spécifique antitumeur. Cette modulation du système immunitaire peut se révéler dangereuse chez l'homme où la détermination de la dose exacte d'immunogène sera difficile à choisir. Néanmoins, elle propose un mécanisme, peutêtre ubiquitaire, de l'immunité tumorale dont la maitrise offre des perspectives prometteuses.

Dans le cas de l'immunité spontanément induite par les tumeurs, il est possible que les HSP relarguées lors de la mort des cellules cancéreuses stimulent le sytème immunitaire par un mécanisme équivalent à celui décrit ci-dessus dans le contexte de vaccination. En outre, si les HSP sont effectivement exprimées à la surface cellulaire, il se pourrait qu'elles agissent elles-mêmes comme des molécules présentatrices d'antigènes à l'instar des molécules du $\mathrm{CMH}$.

\section{Caractéristiques des peptides antigéniques associés aux HSP}

Les séquences des peptides associés aux HSP sont encore inconnues; cependant, dans le cas des tumeurs, on connaît plusieurs mécanismes susceptibles d'entraîner la formation de complexes HSP/peptides tumoraux potentiellement antigéniques, comme la surexpression et l'altération de la conformation de certaines protéines. Ces modifications peuvent être causées par des mutations, comme dans le cas de produits d'oncogène ou d'anti-oncogène dont l'association avec l'hsp70 a été décrite [24]. A titre d'exemple, dans le cancer du sein, la réponse anti- 


\section{RÉFÉRENCES}

26. Schild H, Rötzscgke (), Kalbacher H, Rammensee HG. Limit of T cell tolerance to self proteins by peptide presentation. Science 1990 ; 247: 1587-7.

27. Itzkowitz S. Carbohydrates changes in colon carcinoma. APMIS Suppl 271992 ; $100: 173-80$

28. Di Palma M, Tsamas A, Sidi J, et al. La thermothérapie en cancérologie: place actuelle et perspectives. Cancérologie aujourd'hui 1992 ; 1 : 19-26.

29. Kimura E, Enns RE, Alcaraz JE, Arboleda J, Slamon DJ, Howell SB. Correlation of the survival of ovarian cancer patients with mRNA expression of the $60-\mathrm{kD}$ heat shock protein hsp-60. J Clin Oncol 1993 . $11: 891-8$

30. Ciocca DR, Clark GM, Tandon AK Fuqua SAW, Welch WI, McGuire WL. Hea shock protein hsp70 in patients with axillary lymph node-negative breast cancer: prognostic implications. I Natl Cancer Inst 1993 ; 85 : 570-4.

31. Jameel A, Skilton RA, Campbell TA, Chander SK, Coombes RC, Luqmani YA. Clinical and biological significance of hsp89 alpha in human breast cancer. Int J Cancer 1992; 50 : 409-15.

32. Prehn RT, Prehn LM. The autoimmune nature of cancer. Cancer Res 1987 47 : 927-32.

33. Ménoret A, Meflah K, Le Pendu J. Expression of the $100 \mathrm{kDa}$ glucose regulated protein (grpl(0)/endoplasmin) is associated to tumorigenicity in a model of rat colon carcinoma. Int / Cancer 1994; 56 : 400-5.

34. Sanders RS, Thomas JA, Fina M Germa Z, Benjamin I. Human heat shock protein 70 (hsp70) protects murine cells from injury during metabolic stress. I Clin Invest 1993; 92 : 503-8.

35. Jããttelã M, Wissing $\mathrm{D}$, Bauer PA, Li GC. Major heat shock protein hsp70 protects tumors cells from tumor necrosis factor cytotoxicity. EMBO J 1992; 11 : 3507-12.

36. Huot J, Roy G, Lambert H, Chrétien $\mathrm{P}$, Landry I. Increased survival after treatment with anticancer agents of chinese hamster cells expressing the human $\mathrm{Mr}$ 27.000 heat shock protein. Cancer Res 1991 51: 5245-52.

37. Sierra-Riviera E, Voorhees GJ, Freeman M. Gamma irradiation increases hsp70 in chinese ovary cells. Rad Res 1993; 135 : 40-5.

38. Pechan PM. Heat shock proteins and cell proliferation. FEBS Lett 1991; 280 : 1-4. corps anti-p53 dépend de la formation de complexes particulièrement stables entre la p53 mutée et l'hsp70 [25]. Par ailleurs, une réponse proliférative spécifique de la p53 peut être obtenue avec les lymphocytes de certaines patientes mais les peptides responsables ne sont pas encore identifiés.

On sait qu'il existe des CTL, spécifiques d'antigènes du soi, tolérants dans la mesure où l'antigène est présenté en faible densité par le $\mathrm{CMH}$ de classe I, mais activés lorsqu'il est présenté de manière non physiologique, en forte densité [26]. Une association entre HSP et peptides du soi en quantité anormalement élevée entraînerait une présentation accrue de ces peptides par les molécules du CMH. De nombreuses protéines sont surexprimées et constituent de ce fait des marqueurs tumoraux. Une fraction de ces protéines pourrait donc s'associer aux HSP et engendrer des peptides antigéniques qui n'étaient normalement présents qu'à un niveau relativement bas. Des protéases endogènes, synthétisées en quantité anormale par la cellule tumorale, pourraient produire des fragments protéiques anormaux, donc "non-soi ", qui s'associeraient avec des HSP. En outre, des défauts de maturation des protéines, en particulier de glycosylation, ont été décrits dans les tumeurs [27]. Ceuxci peuvent entraîner un repliement incorrect des protéines concernées qui seront alors chaperonnées par des HSP. Enfin, les cellules tumorales se développent souvent dans des conditions de stress métabolique lié à une faible concentration en $\mathrm{O}_{2}$ ou en glucose, et à un $\mathrm{pH}$ acide. Ces conditions ont pour conséquence une dénaturation des protéines qui seront alors fixées par les HSP.

\section{Résultats cliniques}

L'immunisation avec des HSP de tumeur protège les souris d'une greffe ultérieure de cette même tumeur, mais qu'en est-il chez l'homme? Des traitements équivalents n'existent pas chez les patients. Dans le cas de la thermothérapie, qui devrait stimuler la synthèse
d'HSP, des résultats encourageants ont été obtenus concernant principalement des lésions tumorales superficielles. Ces résultats pourraient être dus à l'effet cytotoxique de la chaleur [28], ou bien à la stimulation d'une réponse immunitaire relayée par les HSP. Il est également possible de corréler le taux de survie des malades avec l'expression de différentes HSP. Dans les cancers ovariens, un taux d'hsp 60 élevé est un facteur prédictif défavorable [29]. Il en est de même pour l'hsp70 dans les cancers du sein non métastasiés [30]. Par analyse différentielle d'une banque d'ADN complémentaire de tumeur du sein hybridée avec un sérum préparé contre des membranes déglycosylées de métastases humaines, le gène de l'hsp90 a été identifié comme un marqueur de tumorigénicité significativement surexprimé dans les métastases de sein [31].

Une forte expression d'HSP dans les tumeurs serait donc un facteur pronostique défavorable, en accord avec l'hypothèse proposée par Prehn que l'existence d'une réponse immune spécifique antitumeur serait un élément facilitant le développement tumoral [32]. Des expériences sur des tumeurs induites chimiquement chez la souris montrent, en effet, que l'immunosuppression inhibe la croissance des tumeurs faiblement immunogènes mais favorise celle des tumeurs qui le sont fortement. En d'autres termes, les tumeurs faiblement immunogènes auraient un développement facilité par une réponse immune importante. Mais il n'est pas certain que cette hypothèse puisse être généralisée à la majorité des tumeurs spontanées qui sont faiblement immunogènes.

L'association entre expression forte d'HSP dans les tumeurs et pronostic défavorable pourrait cependant être due au rôle non immunitaire des HSP dans les cellules stressées: les cellules exprimant fortement les HSP pourraient être plus à même de résister aux agressions antitumeur. Les conditions de microenvironnement du tissu tumoral - vascularisation incomplète, anoxie, $\mathrm{pH}$ acide, manque de nutriments sont des inducteurs de la grp 94/100 dont la surexpression est $\mathrm{m} / \mathrm{s} \quad n^{\circ} 6-7 \mathrm{vol}$. 10, juin-juillet 94 
associée à une forte tumorigénicité [33] ; l'hsp70 est également un protecteur cellulaire lors de l'ischémie [34]. Il a été montré que l'hsp70 et l'hsp27 sont respectivement des protecteurs endogènes efficaces contre l'action du TNF [35] et de certaines drogues chimiothérapiques [36]. L'efficacité de la radiothérapie est peut-être affectée par ces molécules puisque l'irradiation gamma est un inducteur d'HSP [37]. De même, la surexpression des HSP dans des cellules dont la régulation mitotique est affectée n'est pas surprenante dans la mesure où elles sont naturellement impliquées dans les mécanismes liés à la prolifération cellulaire [38]. Ainsi, l'association de l'hsp90 avec les récepteurs des stéroïdes $(\mathrm{m} / \mathrm{s}$ $n^{\circ} 10$, vol. 6, p. 1022) joue un rôle crucial dans la prolifération des tumeurs du sein et de l'ovaire. L'association d'HSP avec des produits d'oncogènes ou d'antioncogènes mutés peut augmenter leur demi-vie et aggraver alors leur pouvoir transformant en fonction du taux d'HSP présentes dans la cellule [24].

L'augmentation de la résistance au stress et de la prolifération des cellules par les HSP, d'une part, et l'immunogénicité des complexes HSP/peptides tumoraux, d'autre part, sont des phénomènes opposés dont l'équilibre peut conditionner, soit le développement, soit la régression des tumeurs.

\section{Conclusion}

La compilation des résultats expérimentaux montre que les molécules chaperons, protéines ubiquitaires, s'avèrent être des acteurs importants de la réponse antitumorale. En tant que porteurs de peptides, elles peuvent participer à la présentation antigénique à des lymphocytes régulateurs ou effecteurs. L'autoimmunité anti-HSP amplifierait la réponse immune spécifique dirigée contre les peptides tumoraux fixés par les HSP. Aussi l'utilisation d'HSP pourrait permettre de stimuler efficacement l'immunité antitumorale. Les molécules chaperons représentent, en outre, un outil remarquable pour caractériser de nouveaux antigènes tumoraux

\section{TIRÉS A PART}

J. Le Pendu.

\section{Summary}

Heat shock proteins and tumor antigens

Heat shock proteins (HSP) belong to the family of chaperone molecules. They associate with peptides or misfolded proteins. Their role as peptide binders and as transporters of these peptides to MHC molecules, as well as the presence of constitutive anti-HSP immune responses, suggest an important role for these proteins in cellular immunity. In tumor cells, HSPs are of ten overexpressed and associated with «abnormal» proteins which are potentially antigenic. Immunisation against some HSPs (grp94, hsp70) purified from some tumors protects animals from a challenge with the tumor from which they were purified. This protection is dependent on the association between HSP and immunogenic peptide(s). However, the nature of these peptides is still unknown and the mechanisms of the HSP-mediated antitumor response are not yet fully understood. 